$9-7-2021$

\title{
Atención de la salud sexual y reproductiva de mujeres migrantes: Un mapeo de actores de la sociedad civil en Baja California, México-2020
}

Silvana Larrea-Schiavon

Population Council

Lucía Vázquez Quesada

Population Council

Esperanza Basurto-Alcalde

Natalia Polgovsky

Isabel Vieitez Martínez

Population Council

See next page for additional authors

Follow this and additional works at: https://knowledgecommons.popcouncil.org/departments_sbsr-rh How does access to this work benefit you? Let us know!

\section{Recommended Citation}

Larrea-Schiavon, Silvana, Lucía Vázquez-Quesada, Esperanza Basurto-Alcalde, Natalia Polgovsky, Isabel Vieitez Martínez, and Pooja Sripad. 2021. "Atención de la salud sexual y reproductiva de mujeres migrantes: Un mapeo de actores de la sociedad civil en Baja California, México-2020." México: Population Council. 


\section{Authors}

Silvana Larrea-Schiavon, Lucía Vázquez Quesada, Esperanza Basurto-Alcalde, Natalia Polgovsky, Isabel Vieitez Martínez, and Pooja Sripad 


\section{Atención de la salud sexual y}

reproductiva de mujeres migrantes: Un mapeo de actores de la sociedad civil en Baja California, México

\section{0}

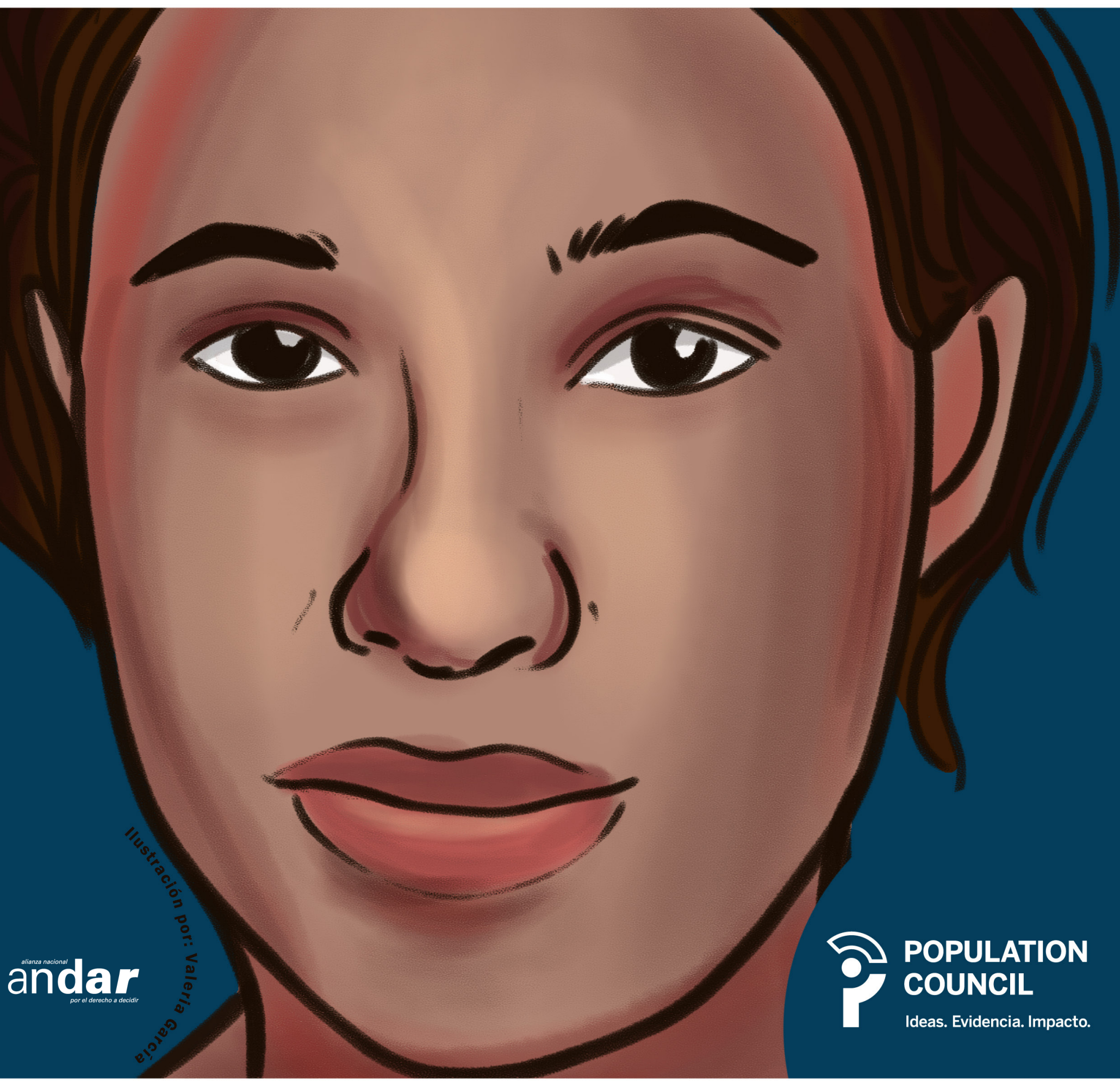




\title{
POPULATION COUNCIL
}

Ideas. Evidencia. Impacto.

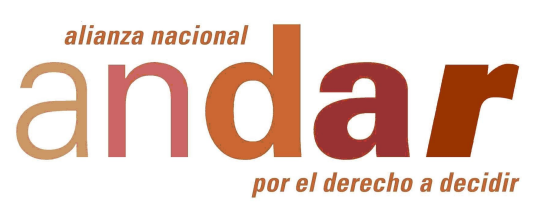

\begin{abstract}
Atención de la salud sexual y reproductiva de mujeres migrantes: Un mapeo de actores de la sociedad civil en Baja California, México. 2020.
\end{abstract}

\section{Equipo de investigación:}

Protocolo de investigación, análisis de datos, redacción y/o revisión de informe:

Silvana Larrea-Schiavon* Lucía Vázquez-Quesada* Isabel Vieitez Martínez*

Pooja Sripad*

*Population Council.

Coordinación del proyecto, recolección y análisis de datos, redacción y revisión de informe:

Esperanza Basurto-Alcalde, consultora.

Recolección de datos, análisis de datos, y redacción de informe:

Natalia Polgovsky-Ezcurra, consultora.

Recolección de datos (personas pasantes y voluntarias):

Arturo Alamilla, entrevistador. Ana Regina Nájera, entrevistadora. Ximena Nateras, entrevistadora. Iván Coria, entrevistador. Gemma Hernández, entrevistadora.

\section{Corrección de estilo:}

Claudia Martínez.

\section{Ilustración y diseño editorial:}

Valeria García.

\section{Agradecimientos:}

Agradecemos el esfuerzo realizado por el equipo de investigación, sobre todo a personas pasantes y voluntarias, así como la gran disposición que las personas entrevistadas tuvieron para compartirnos su experiencia, en pleno inicio de la pandemia causada por el virus SARS-CoV-2, y por su compromiso constante con las mujeres migrantes y otras poblaciones en movilidad.

\section{Aviso legal:}

Se permite la reproducción total o parcial de esta publicación para fines educativos, de investigación y cualquier otro fin libre de lucro, siempre y cuando se cite la fuente.

\section{Cita sugerida:}

Larrea-Schiavon Silvana, Vázquez-Quesada Lucía, Basurto-Alcalde Esperanza, Polgovsky Natalia, Vieitez Isabel, and Sripad Pooja. 2021. "Atención de la salud sexual y reproductiva de mujeres migrantes: Un mapeo de actores de la sociedad civil en Baja California, México. 2020.” México: Population Council. 


\section{HALLAZGOS PRINCIPALES}

- Baja California, específicamente por los municipios de Tijuana y Mexicali, es el principal punto de entrada hacia Estados Unidos para las personas migrantes indocumentadas.

- Se entrevistó a un total de 28 organizaciones de la sociedad civil (OSCs), 16 ubicadas en Tijuana, 10 en Mexicali y 2 en Tecate. Del total, 13 son albergues, 10 organizaciones de la sociedad civil, 1 organización internacional y 4 colectivas independientes.

- La canalización y referencia son los servicios más ofertados por las organizaciones entrevistadas (93\%). El servicio que menos se oferta es la difusión de información (50\%).

- El tipo de servicio directo que se brinda con mayor frecuencia es la atención a sobrevivientes de violencia sexual (57\%) y el que menos, la atención del parto (7\%).

- Las barreras de acceso más mencionadas fueron la falta de recursos económicos, la ubicación lejana de los centros salud, la falta de insumos en las unidades de salud y organizaciones de la sociedad civil y la xenofobia, discriminación y racismo hacia la población migrante.

- La pandemia de COVID-19 ha afectado el acceso a los servicios de salud sexualy reproductiva (SSyR) potencializando barreras previamente existentes como la saturación y falta de servicios, limitándola a la atención de partos.

- La sensibilización y concientización de los servidores públicos y personal de la salud para la atención de las mujeres migrantes se manifestó como una clara y urgente necesidad de cambio para disminuir las barreras de acceso a la salud sexual y reproductiva.

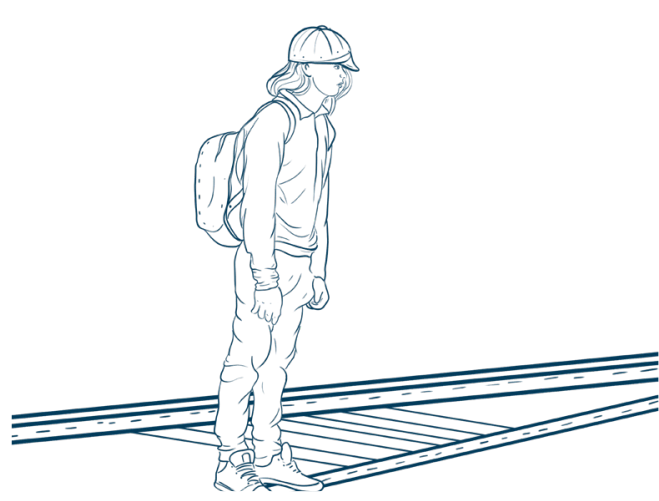

Nota de campo Definitivamente a lo largo de nuestro país son diferentes las situaciones de las migrantes. Particularmente Tijuana ha sido punta de lanza

-Albergue, Baja California

\section{INTRODUCCIÓN}

El estado de Baja California se localiza en la región noroeste de México y comparte 265 kilómetros de frontera con Estados Unidos. El estado se encuentra integrado por cinco municipios: Ensenada, Mexicali, Tecate, Tijuana y Playas de Rosarito (Inafed, s/f). Con base en datos de la Unidad de Política Migratoria, Registro e Identidad de las Personas (UPMRIP) de la Secretaría de Gobernación de México, de enero a julio de 2019 y 2020, 34.3\% de las personas migrantes rescatadas por los Grupos Betas en territorio mexicano sucedió en Baja California, ocupando el primer lugar en el país (UPMRIP, 2020). Por otro lado, 22\% de las personas migrantes orientadas por los Grupos Beta también ocurrió en Baja California (UPMRIP, 2020).

En el Plan Estatal de Desarrollo de Baja California 20202024, se incluyen varias estrategias relacionadas con migración y salud para la población migrante extranjera: 1) promover la firma de convenios de colaboración con las dependencias gubernamentales para que las personas migrantes tengan acceso a la salud; 2) fortalecer la atención de emergencias en salud, con énfasis en riesgos de brotes epidemiológicos en grupos de migrantes y la red de vigilancia de sanidad internacional; 3) acercar los servicios de salud a través del Programa Fortalecimiento a la Atención Médica en los sitios de origen, tránsito y destino de la población migrante; 4) promover la colaboración interinstitucional para el fortalecimiento del Modelo Integral de Atención a la Salud para la población en condición de migración; y, 5) capacitar al personal en desarrollo de competencias culturales en salud y brindar orientación a personas migrantes y sus familiares para la utilización de servicios de salud (Gobierno del Estado de Baja California, 2020).

Baja California reconoce el aborto legal por cuatro causales: violación, riesgo para la vida de la mujer, inseminación artificial no consentida y aborto imprudencial. Aún cuando en el estado se haya aprobado una reforma constitucional para proteger la vida desde el momento de la concepción, las cuatro causales siguen siendo vigentes (Andar, 2020).

\section{OBJETIVO Y METODOLOGÍA}

El presente estudio se enfocó en identificar si las organizaciones de la sociedad civil brindan los servicios de difusión de información, consejería, canalización, acompañamiento y atención directa a mujeres migrantes en tránsito por México en temas referentes a métodos anticonceptivos, anticoncepción de emergencia, interrupción del embarazo, pruebas de embarazo, atención prenatal, 
atención del parto, atención del postparto, salud menstrual, infecciones de transmisión sexual (ITS) y atención a sobrevivientes de violencia sexual.

Para alcanzar dicho objetivo se hizo una búsqueda dirigida de organizaciones que trabajaran en temas de SSyR y/o con mujeres migrantes en siete estados claves en la ruta migratoria en la República mexicana: Chiapas, Tabasco, Veracruz, Oaxaca, Ciudad de México, Chihuahua y Baja California. Se identificó a un actor clave dentro de cada organización y se realizó una entrevista telefónica semiestructurada (para más información ver el reporte general del proyecto). En este documento se presentan los resultados para el estado de Baja California.

\section{RESULTADOS}

\section{Perfil de las organizaciones entrevistadas}

Por búsqueda dirigida se identificó un total de 69 organizaciones que cumplían con los criterios de inclusión, de las cuáles se logró entrevistar a 28 (40.5\%): 13 albergues y refugios, y 15 organizaciones de la sociedad civil. Asimismo, 17 organizaciones cuentan con trabajo a nivel local (60.7\%), 5 a nivel nacional (17.8\%), 3 a nivel binacional (10.7\%) y 3 son organizaciones internacionales (10.7\%). Del total, 7 son de carácter religioso (25\%) 16 organizaciones se autodefinen como laicas (57.1\%) y 5 como feministas o parte del movimiento feminista (17.8\%). Las organizaciones entrevistadas se encuentran localizadas en tres municipios: Tijuana ( $n=16)$, Mexicali $(n=10)$ y Tecate $(n=2)$ (ver Gráfica 1).

A su vez, 7 organizaciones brindan atención especializada a mujeres y adolescentes de las cuales 1 corresponde a un albergue, 2 son OSCs y 4 son colectivas independientes. Las 21 organizaciones restantes atienden a población en general, es decir a mujeres y hombres, adolescentes, niñas y niños, e incluso a recién nacidos.

\section{Servicios brindados para la atención de la SSyR de las mujeres migrantes}

Del total de organizaciones entrevistadas en Baja California, 14 (50\%) difunden información, 20 (71.4\%) dan consejería, 26 (92.8\%) canalizan casos, 15 (53.5\%) acompañan ${ }^{1}$ a las mujeres durante la atención y 20 (71.4\%) brindan atención directa en alguno de los temas mencionados.

Es importante destacar para el caso de Baja California la presencia de colectivas independientes que, aunque no se
Gráfica 1. Porcentaje de organizaciones con base en el municipio donde trabajan. Baja California, 2020.

7.1

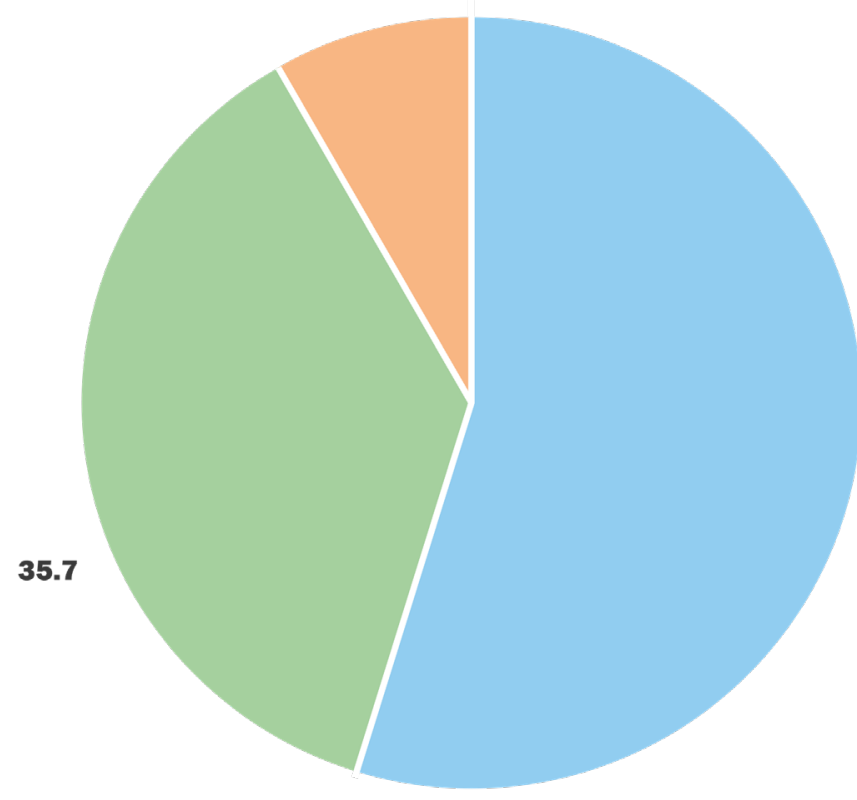

57.1

Tijuana

Mexicali

Tecate

enfocan al trabajo con población migrante, han brindado asesoría y acompañamiento a mujeres migrantes, sobre todo en el tema de acceso a un aborto seguro. La labor de estas colectivas se enfoca en población de mujeres y adolescentes específicamente en la guía y acompañamiento del acceso a un aborto seguro.

Cuando se hace el cruce entre tipo de servicio (difusión de información, consejería, atención directa, canalización y referencia y acompañamiento) y temática de SSyR en la cual se ofertó este servicio se identificó que menos del 36\% de las organizaciones entrevistadas brindó alguno de los servicios para la mayoría de las condiciones de SSyR, aún cuando existe un mayor número de OSCs en Baja California en comparación con otros estados incluidos en el estudio. Los principales temas sobre los cuales se difundió información fueron los métodos anticonceptivos y la atención a sobrevivientes de violencia sexual. La consejería se dio principalmente en el tema de la atención a sobrevivientes de violencia sexual. La atención directa brindada por las organizaciones se concentró alrededor de métodos anticonceptivos y atención a sobrevivientes de violencia sexual. La canalización y referencia se brindó por más del 36\% de las organizaciones para todos los temas, excepto para anticoncepción de emergencia, atención del postparto y salud menstrual. Por último, el

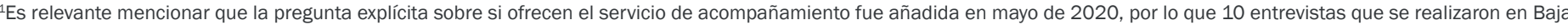


acompañamiento fue el servicio menos provisto por las OSCs entrevistadas, donde menos del 36\% ofreció este servicio en todos los temas, excepto para la atención a sobrevivientes de violencia sexual (50\% de las organizaciones identificaron sí dar acompañamiento). 5 de las 28 organizaciones entrevistadas dieron atención directa para informar sobre la interrupción del embarazo y 6 mencionaron dar atención directa para anticoncepción de emergencia. Asimismo, más de la mitad de las organizaciones dieron este servicio para las mujeres sobrevivientes de violencia sexual (ver Tabla 1).
Los métodos anticonceptivos que se brindaron con mayor frecuencia son los preservativos masculinos y femeninos. Algunas organizaciones más especializadas y que cuentan con más recursos también ofrecieron métodos inyectables, anticoncepción oral y, específicamente las que trabajan con trabajadoras sexuales, lubricantes a base de agua. Si la mujer solicita otro medio anticonceptivo se le canaliza a otras organizaciones o unidades de salud. Quienes difundieron información sobre el tema lo hicieron a través de folletos, pláticas y talleres. Con respecto a las pruebas de embarazo,

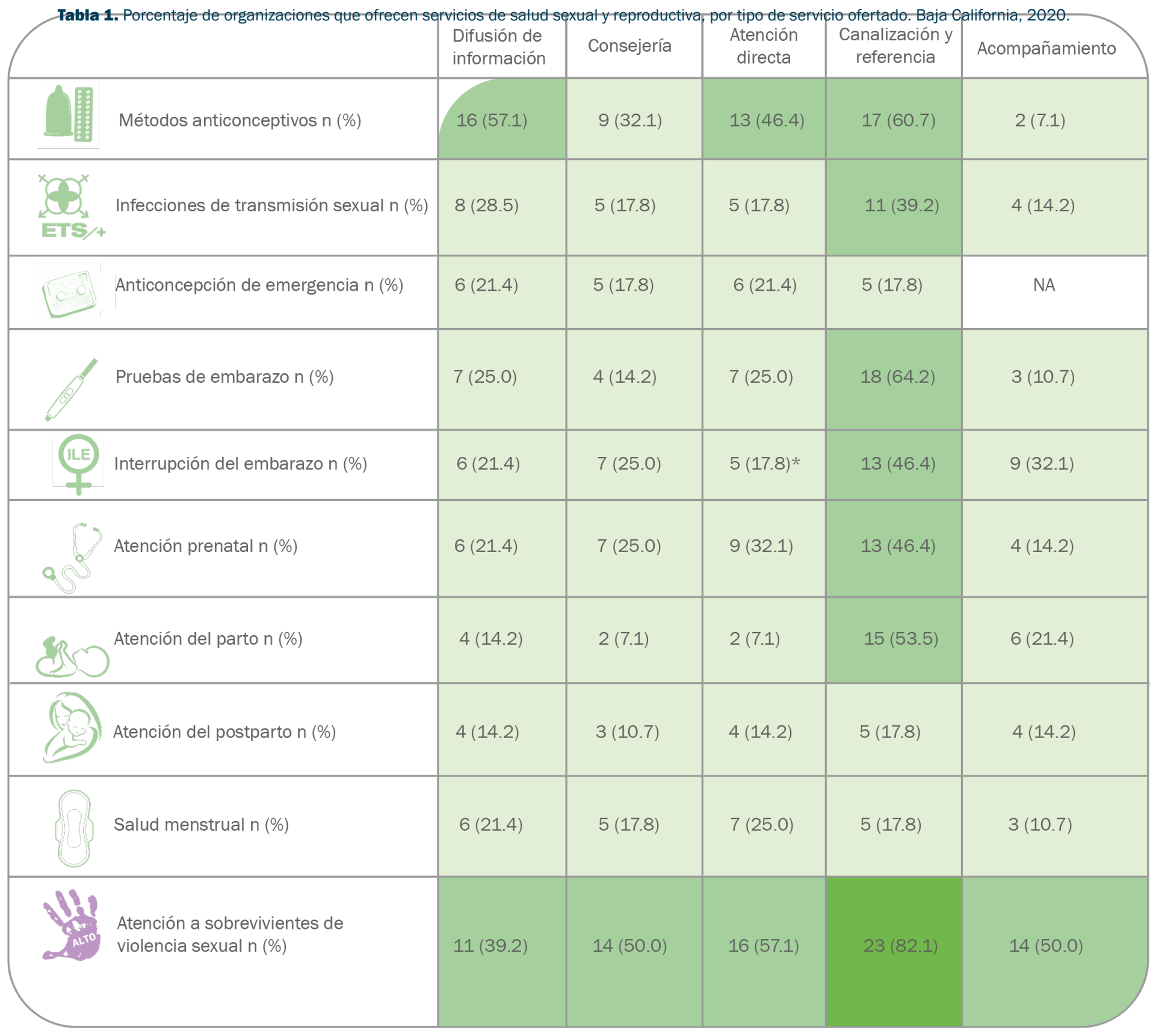

Nota: verde obscuro, el servicio es ofertado por $>70 \%$ de las organizaciones entrevistadas; verde medio, el servicio es ofertado por entre $36 \%$ - $69 \%$ de las organizaciones entrevistadas; verde claro, el servicio es ofertado por $<36 \%$ de las organizaciones entrevistadas.

* La atención directa en el tema de interrupción del embarazo consiste en brindar información pública amplia sobre el marco legal (particularmente de la NOM-046), los métodos recomendados por la Organización Mundial de la Salud, y los requisitos para acceder a un servicio legal. Las OSCs no realizan los procedimientos, ni promueven la interrupción del embarazo - pues realizarlo es decisión de la mujer embarazada y de quien ella quiera involucrar al tomarla-. Se diferencia de "difusión de información" en tanto los temas cubiertos son más amplios en esta categoría. 
las organizaciones canalizan a las mujeres a farmacias y laboratorios económicos. Si la mujer quiere interrumpir el embarazo, algunas de las organizaciones aconsejan también realizar un ultrasonido para conocer las semanas de gestación en laboratorios económicos. Para el caso de la salud menstrual, algunas OSCs dieron pláticas sobre el ciclo menstrual, el cuerpo-territorio, el uso de copas y la higiene menstrual.

Sobre la interrupción del embarazo, las colectivas son las que más trabajan con este tema. Realizaron acciones de difusión de sus teléfonos de contacto y los servicios de información sobre aborto legal y seguro que brindan, repartiendo folletos cerca de las maquilas, en supermercados y en las estaciones de autobús y con lonas en el muro de la frontera y en las calles. Además, ofrecieron información telefónica sobre este tema. Cuando se requiere, canalizan a las mujeres a la Ciudad de México.

Para la atención del parto, se mencionó que en Tijuana hay una fuerte presencia de parteras que han sido clave en la atención de partos en la población de mujeres migrantes. Las parteras también apoyan visitando algunas organizaciones. En otros casos, se canaliza a las mujeres al Hospital General y al Hospital Materno-Infantil.

Las organizaciones especializadas en salud o que trabajan con la comunidad LGBTIQ+ son las que principalmente difundieron información sobre las ITS. Además, ofrecieron preservativos masculinos y femeninos, y jeringas para las personas usuarias de drogas inyectables. Algunas también realizaron pruebas rápidas para la detección de VIH, sífilis, hepatitis y clamidia. Las organizaciones que se especializan en dar atención en salud orientan, asesoran y acompañan a las personas migrantes $\mathrm{VIH}+$ en la realización de los trámites necesarios para darse de alta en los CAPASITS y poder acceder gratuitamente al tratamiento antirretroviral. Solamente 1 de las organizaciones entrevistadas ofrece la vacuna contra el virus del papiloma humano (VPH).

Por último, la mayoría de las organizaciones buscó dar atención psicológica a las mujeres migrantes sobrevivientes de violencia sexual. En caso de no contar con el personal para brindar esta atención, canalizan a las mujeres. Las organizaciones con mayores recursos y especializadas en atención de la salud ofrecen profilaxis postexposición, profilaxis para la prevención de otras ITS y anticoncepción de emergencia. Existe un constante acompañamiento en el estado para el levantamiento de denuncias y los trámites jurídicos.

Cuando observan que no pueden dar la atención directa, las organizaciones recurren a la canalización hacia otras organizaciones, al sistema de salud público o a médicos y consultorios particulares, no obstante, las posibilidades de atención dependen en cierta medida de la presencia de personal de salud en la organización. De las 28 organizaciones entrevistadas en Baja California, 10 indicaron que no cuentan con ningún personal de salud en sus instalaciones, 10 tienen personal médico de base, 5 cuentan con un personal médico itinerante, 2 con la presencia de otro tipo de profesionales de la salud y 1 con personal médico voluntario (ver Gráfica 2).

Gráfica 2. Porcentaje de organizaciones que cuentan con personal de salud en sus instalaciones. Baja California, 2020.

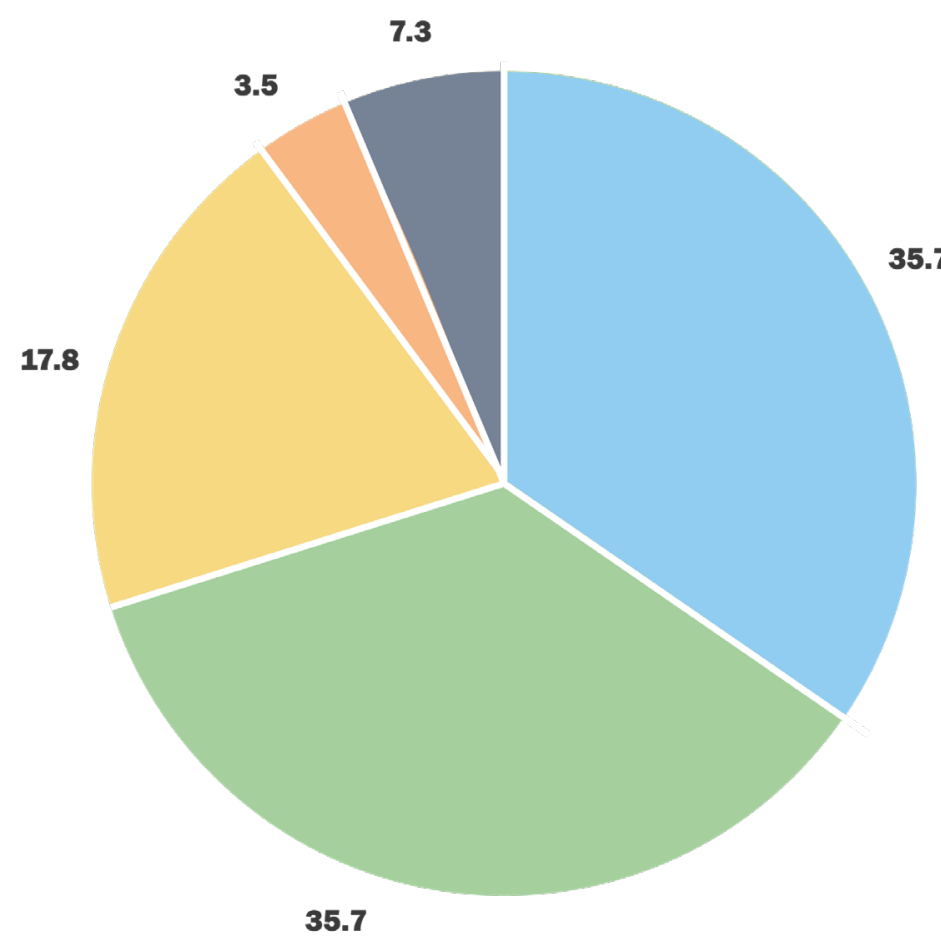

Sin personal de salud

Médica/o de base

Médica/o itinerante

Médica/o voluntaria/o

Otros/as profesionales de la salud

\section{Principales barreras para la atención en} SSyR a mujeres migrantes

En las entrevistas con organizaciones de la sociedad civil en Baja California se identificaron múltiples barreras para que las mujeres migrantes pudieran acceder a los servicios de SSyR durante su estancia o tránsito por el estado. En el caso del presente estudio, las barreras para el acceso a la salud fueron clasificadas en cuatro categorías: 
1. Normativas: relacionadas con la legislación, políticas y normatividad que aplican tanto a la población migrante (marco restrictivo para la atención), como a las instituciones de salud;

2. Institucionales: impedimentos estructurales de la institución de salud que brinda o debería brindar los servicios;

3. Organizacionales: impedimentos estructurales de la organización de la sociedad civil que brinda los servicios; e

4. Individuales: obstáculos derivados de la condición socioeconómica, cultural y subjetiva de las personas migrantes (ver Tabla 2).

Se identificaron dos barreras normativas en las entrevistas con las organizaciones de la sociedad civil. La primera fue el cambio constante en la administración pública (cada tres años para el municipio de Tijuana), lo cual genera dificultad para dar continuidad a las alianzas, acuerdos, convenios, apoyos y proyectos establecidos y en curso. Esto obliga a las organizaciones a acercarse a la nueva administración, explicar el trabajo que realizan y negociar la posibilidad de continuar con las alianzas, convenios, apoyos y proyectos previos. La segunda barrera se relaciona con la normativa estatal relativa a la interrupción del embarazo, especialmente reflejada en obstáculos legales para poder acceder al servicio. Algunas organizaciones prefirieron no hablar del tema y otras mencionan que este miedo también se presenta en las mujeres migrantes. Por este motivo, en caso de que la mujer solicite la interrupción del embarazo, las organizaciones la canalizan a otras OSCs, ya que consideran que no se puede contar con las unidades de salud.

Se identificó que los obstáculos existentes en las instituciones de salud afectan a toda la población de Baja California, pero en particular a las mujeres migrantes ya que, por el simple hecho de ser migrantes extranjeras, sufren discriminación y racismo por parte del personal de salud (debido a que hablan otro idioma o a que tienen otra tonalidad o color de piel). Las barreras institucionales mencionadas por las organizaciones fueron: 1) la falta de insumos, principalmente medicamentos; 2) la ubicación geográfica de los centros de salud, particularmente en Tijuana, ya que están lejos del centro donde se localizan la mayoría de las organizaciones y albergues para migrantes; 3) la falta de difusión de programas de atención para población migrante entre ésta y el propio personal de salud; 4) la saturación de los servicios; 5) desconocimiento del personal de salud sobre la política de atención a población migrante, por lo cual les solicitan documentos para brindarles la atención ${ }^{2}$; 6) la falta de personal de salud capacitado y sensibilizado, incluyendo a traductoras y traductores; 7) la falta de reconocimiento por parte del personal de salud sobre la importancia y trabajo que las parteras tradicionales realizan en el estado; 8) rotación constante del personal de salud; 9) desconocimiento e incumplimiento de la NOM-046, pues el personal de salud cuestiona insistentemente a las mujeres migrantes sobre su procedencia, sus motivos para migrar y las condiciones en su país, cuando éstas solicitan la interrupción del embarazo; 10) procesos lentos y prolongados para la denuncia de violencia sexual, tiempo con el que muchas veces las mujeres no cuentan; y, 11) falta de disponibilidad de personal médico femenino para la atención de mujeres víctimas de violencia sexual -lo cual las obliga a ser atendidas por hombres-.

Las barreras organizacionales identificadas por las OSCs fueron 1) la falta de recursos e insumos médicos, 2) la ausencia de personal de salud especializado en sus instalaciones y 3) la infraestructura de las organizaciones, lo cual no les permite brindar una atención más integral a las mujeres migrantes.

Por último, las barreras individuales para el acceso a los servicios de SSyR para las mujeres migrantes mencionadas por las OSCs entrevistadas fueron: 1) la falta de recursos económicos para transportarse hasta las unidades de salud, para comprar medicamentos y/o para realizarse estudios de laboratorio y de imagen; 2) desconocimiento sobre la ubicación de las unidades de salud, los requisitos para acceder a la atención y sobre su derecho al acceso a los servicios de salud; 3) el miedo a ir a las unidades de salud, debido a experiencias previas de discriminación y malos tratos por parte del personal de salud y otros servidores públicos, así como a ser reportadas a las autoridades migratorias; 4) pocas o nulas redes de apoyo que le faciliten información sobre SSyR y que le animen a priorizar su salud por el contrario, si las mujeres se encuentran en una relación

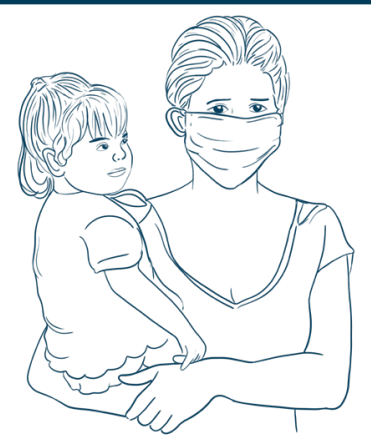

Nota de campo Aún sabiendo que una emergencia debe ser atendida independientemente de la condición migratoria de una persona, los servidores de los hospitales generales les dan información errónea, las rechazan, mandándolas a diferentes centros de salud, negándoles así la atención

- Organización, Baja California 
caracterizada por el machismo, la búsqueda de información sobre SSyR puede verse afectada por miedo, por ejemplo, a que su pareja o acompañante no esté de acuerdo; 5) el idioma como una limitante importante, dado que una gran parte de las mujeres migrantes no hablan de español, ya que vienen de comunidades indígenas de países de Centroamérica, de Haití, Ghana y Camerún; y, 6) la presencia de estigmas culturales y tabúes sobre la sexualidad y la interrupción del embarazo (muchas mujeres provienen de países sumamente religiosos).

\section{COVID-19, mujeres migrantes y acceso a} servicios de SSYR

En general, y de acuerdo con el reporte de las organizaciones entrevistadas en los siete estados que son parte de este estudio $^{3}$, se identificó que la pandemia por COVID-19 tuvo los siguientes efectos en las OSCs y en la población migrante, particularmente en las mujeres y su acceso a servicios de
SSyR:

- Reducción de unidades de salud y servicios disponibles. La reconversión de clínicas y hospitales a Centros COVID y la atención restringida a casos de emergencia, limitó de manera significativa la atención en las unidades médicas, ya sea por retraso o negación de la atención.

- Cierre temporal y/o parcial de albergues. Incluso aquellos que se mantuvieron abiertos dejaron de recibir a personas, ya que las y los migrantes no acudían porque escucharon que estaban cerrados.

- Disminución de recursos en las OSCs para prestar la atención. Con la pandemia hubo una reducción de recursos recibidos a través de donativos, además de tener una baja en el número de personas voluntarias, $\mathrm{y}$ del personal en general para brindar servicios y atención, incluyendo a personal médico.

Tabla 2. Resumen de principales barreras para que las mujeres migrantes accedan a los servicios de salud en Baja California, 2020.

\begin{tabular}{|c|c|}
\hline Clasificación & Principales barreras \\
\hline Normativas & $\begin{array}{l}\text { - Cambio constante de la administración pública } \\
\text { - Penalización del aborto en el estado }\end{array}$ \\
\hline Institucionales & $\begin{array}{l}\text { - Falta de insumos principalmente medicamentos } \\
\text { - Ubicación geográfica } \\
\text { - Falta de difusión de programas de atención para migrantes } \\
\text { - Saturación de los servicios } \\
\text { - Desconocimientos del personal de salud } \\
\text { - Falta de personal sensible y capacitado, incluyendo traductoras y traductores } \\
\text { - Falta de reconocimiento de la partería tradicional } \\
\text { - Rotación constantes de personal administrativo y de salud } \\
\text { - Desconocimiento e incumplimiento de la NOM-046 } \\
\text { - Procesos lentos para la atención de sobrevivientes de violencia sexual } \\
\text {-Falta de personal médico femenino para atender casos de violencia sexual }\end{array}$ \\
\hline Organizacionales & $\begin{array}{l}\text { - Falta de recursos económicos } \\
\text { - Falta de personal general y especializado } \\
\text { - Falta de infraestructura adecuada para brindar atención más directa e integral }\end{array}$ \\
\hline Individuales & $\begin{array}{l}\text { - Falta de recursos económicos } \\
\text { - Desconocimiento } \\
\text { - Miedo } \\
\text { - Machismo y ausencia de redes de apoyo } \\
\text { - Idioma } \\
\text { - Concepciones distintas, estigmas, tabúes }\end{array}$ \\
\hline
\end{tabular}

${ }^{3}$ En este apartado se incluye información general y que se observó para los siete estados: Chiapas, Tabasco, Veracruz, Oaxaca, Ciudad de México, Chihuahua y Baja California. 
- Disminución de recursos en las OSCs para prestar la atención. Con la pandemia hubo una reducción de recursos recibidos a través de donativos, además de tener una baja en el número de personas voluntarias, y del personal en general para brindar servicios y atención, incluyendo a personal médico.

- Permanencia prolongada de migrantes en los lugares, al no poder seguir su viaje. El cierre de oficinas gubernamentales - como la Comar- afectó a aquellas personas que esperaban la resolución a su solicitud de condición de refugiada u otro trámite similar, y les obligó a no salir de la entidad donde iniciaron el procedimiento.

- Afectaciones a la salud mental de las personas migrantes. Incremento de las preocupaciones relacionadas con sus procesos frente a autoridades migratorias, y/o por el confinamiento en albergues.

- Pérdida de trabajos y recursos económicos. Con el cierre de establecimientos comerciales hubo pérdida de empleos que también afectó a personas migrantes, pues se enfentan a la imposibilidad de laborar y tener el ingreso para solventar sus necesidades básicas.

- Miedo a asistir a las unidades médicas. Las personas migrantes tienen temor a contagiarse si asisten a las unidades médicas, por lo que retrasan o desisten de la atención.

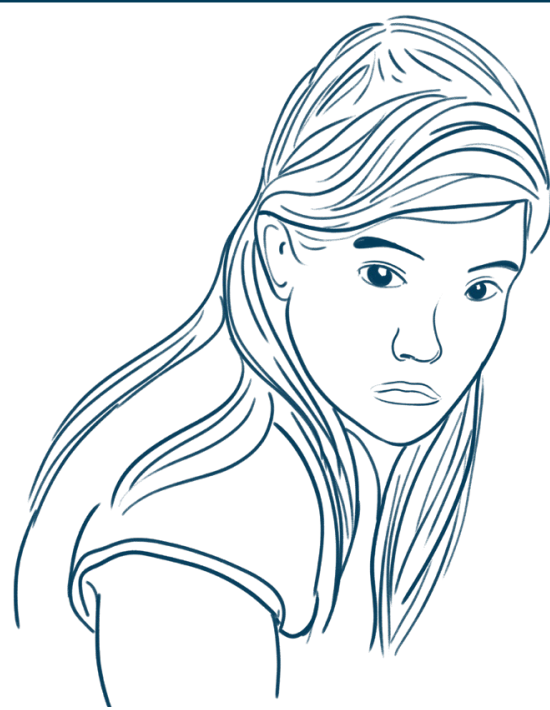

Nota de campo La propaganda del gobierno es que el aborto es un delito y no puede practicarse. En Baja California hay 25 mujeres en la cárcel por homicidio por aborto.

- Organización, Baja California

\section{CONCLUSIONES Y ÁREAS DE OPORTUNIDAD ${ }^{4}$}

Para que las mujeres migrantes indocumentadas y solicitantes de asilo puedan acceder a servicios de salud responsivos a sus necesidades y de calidad en Baja California, es necesaria una perspectiva de sistemas de salud. Esta perspectiva considera que las y los actores relevantes (instituciones, organizaciones y personas) son una misma colectividad en funcionamiento que debe coordinarse para prevenir enfermedades o padecimientos, y atender y promover la salud de esta población. En este sentido, aun cuando la sociedad civil no es responsable de brindar servicios de salud, las organizaciones son clave para contribuir a este propósito.

En el campo en que se enfocó el estudio (SSyR de las mujeres migrantes), el rol de las OSCs puede ser particularmente relevante, no sólo por ser muchas veces el primer punto de contacto, sino porque la confianza que ganan con su trabajo les puede habilitar para tratar estos temas -considerados de mayor intimidad-.

El Estado, a través de sus instituciones, tiene la responsabilidad de garantizar que los servicios de salud se brinden de forma oportuna a las mujeres migrantes. El apoyo a la continua capacitación de servidores públicos sobre los derechos de personas migrantes en México (y sobre lo que implican los cambios en políticas públicas) puede fortalecer la viabilidad de las rutas de acceso a servicios de salud.

Con respecto a los servicios de salud en Baja California, un área de oportunidad importante es que los programas, acuerdos y protocolos para la atención en salud de la población migrante en unidades médicas no se eliminen al término de una administración, sino más bien, se identifiquen áreas de mejora con base en un diagnóstico integral de los mismos. Por otro lado, se recomienda la implementación de variables en sus sistemas de información que permitan hacer diagnósticos periódicos de las necesidades en salud de la población migrante $y$, específicamente, de las mujeres en situación de movilidad. Esto les permitiría redirigir esfuerzos y recursos a las necesidades en salud más apremiantes, además de reforzar la capacitación de su personal en estas temáticas. En caso de que estas variables sean recabadas, la información podría hacerse pública para involucrar a las organizaciones de la sociedad civil en las estrategias de promoción y atención a la salud.

Para reducir las barreras relacionadas con la dificultad geográfica para acceder a una unidad de salud que pueda atender las necesidades de SSyR de las mujeres migrantes, es importante encontrar estrategias viables de alcance

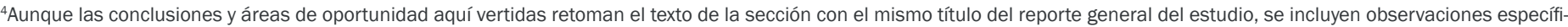
cas para el estado de Baja California. 
comunitario que trasciendan las áreas de promoción de la salud y prevención de enfermedades transmisibles. Por ejemplo, la implementación de unidades de atención de primer nivel móviles que acudan a los albergues y/o puntos de reunión de las mujeres migrantes. Se podría contactar a organizaciones internacionales (ej. Médicos Sin Fronteras) que han implementado este tipo de estrategias para considerar una colaboración o la oportunidad de compartir buenas prácticas con los servicios de salud. La telesalud podría ser otra opción para la promoción y atención a la salud de la población en tránsito.

También se mira necesaria la implementación de una plataforma formal y efectiva de capacitación y sensibilización del personal de salud respecto a legislación y políticas públicas nacionales y estatales referentes a migración y salud, incluida la dimensión más operativa de cómo implementar estas políticas a nivel local; derechos humanos; SSyR de mujeres migrantes, incluida la interrupción del embarazo; y diferencias culturales que existen entre el personal de salud y las mujeres migrantes.

Con la información recolectada a través de las entrevistas, se observó que el estado de Baja California puede ser punta de lanza para demostrar la importancia de integrar a las parteras en la atención integral de la SSyR, sobre todo en poblaciones que históricamente han tenido menor acceso a servicios de salud institucionalizados y occidentalizados, y que pudieran beneficiarse de los conocimientos y la atención brindada por las parteras a lo largo del embarazo y durante el parto, ligando estos servicios con unidades de salud que cuenten con los insumos y el personal necesario para atender embarazos de alto riesgo o complicaciones graves durante el parto.

Por su parte, las organizaciones de la sociedad civil pueden, entre otras cosas, ayudar a monitorear las necesidades de SSyR de esta población; brindar información/educación de calidad sobre la SSyR (con base en las necesidades identificadas), y sobre los pasos para ejercer su derecho a la salud; identificar (y educar también sobre) síntomas y señales de riesgo que indican que una mujer debe de ser atendida de urgencia; y establecer mecanismos formales de referencia a unidades de salud correspondientes con el tema a tratar.

En el caso de Baja California, las áreas de oportunidad identificadas para sí mismas por las organizaciones civiles entrevistadas, se dirigen principalmente a la falta de recursos. Para atender esta área de oportunidad, se recomienda la colaboración con otras OSCs que trabajen con mujeres migrantes, en temas de SSyR o ambas, con la finalidad de sumar esfuerzos y recursos y utilizarlos de manera más eficiente y con la finalidad de beneficiar a esta población. Las organizaciones pueden difundir información preventiva sobre indicios de alarma (cuándo buscar un servicio de salud), contactos de organizaciones que pueden apoyarlas vía remota, e información sobre cómo acceder a servicios de salud en el estado o en otros estados de la ruta migrante. Generar y/o fortalecer la articulación de organizaciones que trabajan para y con migrantes con otras que trabajan estos temas (feministas, por ejemplo) es un primer paso para integrar la atención a la salud de las mujeres migrantes. El diálogo con estas organizaciones será relevante para que la información y requisitos de atención (como ser necesariamente acompañadas) se adecúe a las circunstancias y necesidades de las mujeres migrantes y solicitantes de asilo.

Otra área de oportunidad es la de contar con un sistema de información que les permita el monitoreo de la población a la que atienden y los problemas de salud (en general y en particular, SSyR) presentados $\neg$-entre otras temáticas relacionadas con el respeto a sus derechos humanos-. La finalidad del monitoreo es la de sistematizar e identificar las necesidades más apremiantes de esta población y cómo se van transformando en el tiempo. Esto ayudará a las organizaciones a contar con información para hacer incidencia con las instituciones de salud gubernamentales, y recomendar la priorización de esfuerzos y recursos en el corto, mediano, y largo plazo. El sistema de información se puede generar también con el apoyo de organizaciones de la sociedad civil nacionales y/o internacionales. Con el fin de hacerse de recursos para la creación de este sistema de información seguro y compartido entre las redes de apoyo a migrantes, se puede insistir a través de la incidencia con quienes financian trabajo en este campo.

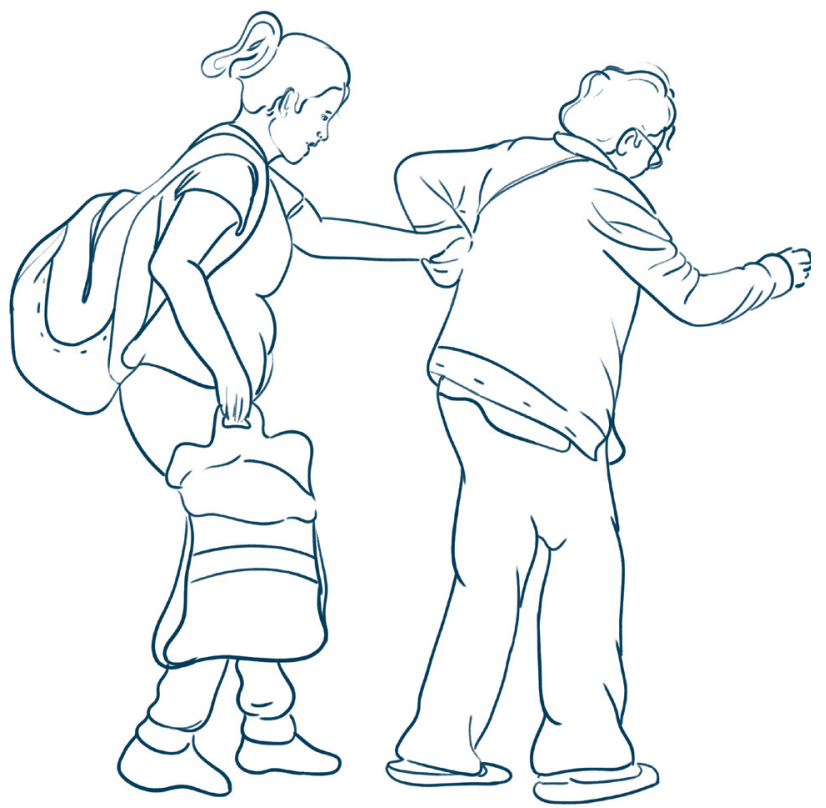


Se identificó que las organizaciones de la sociedad civil perciben que en el estado persiste, tanto por parte del personal de salud como de la población, una actitud de criminalización de la interrupción del embarazo, lo cual aumenta las barreras para acceder a estos servicios, no solo para para las mujeres migrantes sino para las mujeres en general. Se propone para esta área de oportunidad el trabajo de incidencia política para que las autoridades estatales no fomenten la criminalización de la interrupción del embarazos $y$, al contrario, que velen por una mejor SSyR en el estado y por el derecho de las mujeres a vivir una sexualidad libre, placentera y respetuosa, así como poder acceder a servicios de salud que les permitan decidir si, cuándo y cuántos hijos o hijas desean tener.

A pesar de las limitaciones del estudio (como contar sólo con el reporte de las OSCs, y no con la perspectiva ni de las mujeres ni del sistema de salud local), la investigación muestra importantes áreas de oportunidad para lograr la atención a la SSyR de las mujeres migrantes. Investigaciones futuras deberán de indagar directamente sobre las necesidades de SSyR de mujeres, adolescentes y niñas, y las necesidades del sector salud para poder prestar la atención. La investigación operativa puede mejorar el funcionamiento de las OSCs como puentes para acercar a las mujeres a los servicios de salud, y apoyar a las unidades médicas a mejorar sus protocolos, así como a entender por qué ciertos esfuerzos - de capacitación, por ejemplo-, pueden no estar dando el resultado esperado.

La salud sexual y reproductiva de las mujeres migrantes en tránsito por México es un derecho. Tomar conciencia de que las necesidades de salud de las mujeres son minimizadas, incluso por ellas mismas, es uno de los retos hacia la equidad de género. Facilitar y garantizar el cuidado a su salud forma parte de los esfuerzos para lograr el respeto a los derechos humanos.

Agradecemos el esfuerzo realizado por el equipo de investigación durante los meses de trabajo, así como la gran disposición que las personas entrevistadas tuvieron para compartirnos su experiencia en medio de la pandemia de SARS-CoV-2, y por su compromiso constante con las mujeres migrantes.
El presente estudio es evidencia de que las organizaciones de la sociedad civil locales e internacionales trabajan en primera línea para defender, promover, y apoyar el alcance de los derechos de las personas migrantes -incluso coadyuvando en el acceso a servicios de salud sexual y reproductiva-.

\section{El reconocimiento a ellas y a su labor va acompañado de un Ilamado a la solidaridad para impulsar su financiamiento y generar condiciones que permitan su desarrollo y el de sus colaboradoras/es.}

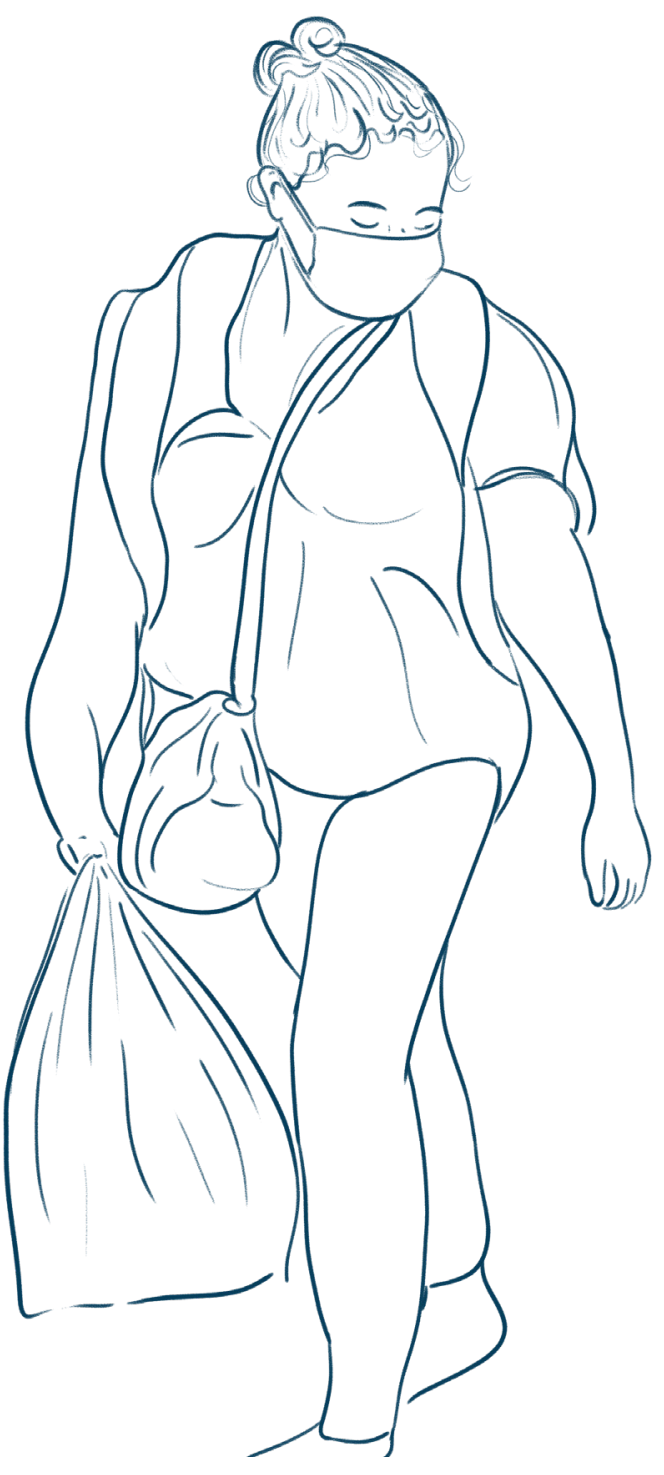

Population Council México. 2021. Atención de la salud sexual y reproductiva de mujeres migrantes: Un mapeo de actores de la sociedad civil en México. Resultados principales para el estado de Chiapas. Ciudad de México, México: Population Council. 
REFERENCIAS:

1. Alianza Nacional por el Derecho a Decidir (Andar). Aborto Legal. Baja California. México, 2020. Disponible en: https://andar. org.mx/aborto-legal/\#BAJACALIFORNIA

2. Gobierno del Estado de Baja California. Plan Estatal de Desarrollo 2020-2024. México, Baja California, 2020.

3. Instituto Nacional para el Federalismo y el Desarrollo Municipal (Inafed). Enciclopedia de los Municipios y Delegaciones de México. Estado de Baja California. S/F. Disponible en: http://www.inafed.gob.mx/work/enciclopedia/EMM02bajacalifornia/ mediofisico.html

4. Unidad de Política Migratoria, Registro e Identidad de las Personas (UPMRIP). Estadísticas Migratorias. Síntesis 2020. Secretaría de Gobernación. México, 2020. 